\title{
Chemistry with Photons, Protons, and Electrons
}

\author{
Jonathan C. Freys, David Hanss, Mathieu E. Walther, and Oliver S. Wenger*
}

\begin{abstract}
This is an account of the research activities of our group during the first two years of its existence. First results from our work on proton-coupled electron transfer and long-range charge tunneling reactions are presented. This includes a hydrogen-bonded cation-anion pair in which a proton-coupled electron transfer process can be phototriggered and followed by simple optical spectroscopic means, as well as a series of rigid rod-like donor-bridge-acceptor molecules which we use to investigate physical phenomena associated with the tunneling of electrons or holes. A unifying feature of this research is the use of light (photons) to induce proton and/or electron transfer.
\end{abstract}

Keywords: Electron transfer - Molecular wires · Photoredox chemistry · Proton-coupled electron transfer . Tunneling

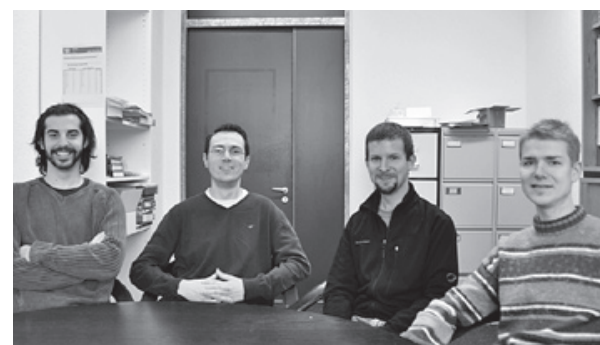

\section{Introduction}

Proton-coupled electron transfer is a process of key importance in biology. For instance, the conversion of water to molecular dioxygen in photosystem II involves a four-electron/four-proton coupled oxidation reaction. ${ }^{[1]}$ Other examples of biologically relevant proton-coupled electron transfer reactions are found in respiration and nitrogen fixation. ${ }^{[2]}$ Many aspects of this class of reactions are poorly understood, which is partly due to the complexity of the involved biological systems. This makes the search for simple model systems for proton-coupled electron transfer reactions an important research target. Our newly created research group has taken on this challenge, and our first results in this area are presented in Section 2.

Controlled electron flow is a requirement for efficient storage and conversion of energy, and it is essential for successful operation of molecular-scale electronic devices. A critical issue is understanding bridge energy effects on charge transport through molecular materials, hence our interest in (phototriggered) hole and electron tunneling through rigid covalent bridges. Of particular interest to us is the investigation of the influence of barrier width and height on the tunneling rates. First results obtained along this line of research are presented in Section 3.

\section{Proton-coupled Electron Transfer}

Under what circumstances is it possible to transfer simultaneously an electron and a proton onto a single substrate? This is one of the key questions that must be answered if we are to activate small inert molecules such as $\mathrm{N}_{2}$ or $\mathrm{CO}_{2}$ by proton-coupled electron transfer. Toward this end, our group has launched a search for simple artificial model systems in which both electron and proton translocations can be monitored by relatively straightforward (optical spectroscopic) means. We have found that the hydrogen-bonded cation-anion pair shown in the shaded area of Fig. 1 possesses the desired properties. ${ }^{[3]}$ The cyclometalated iridium complex forms hydrogen bonds with various benzoate anions in apolar solution and in the solid state. Upon irradiation with blue light, the iridium complex is promoted to a long-lived excited state $(\tau=1.8 \mu \mathrm{s})$ in which it becomes a potent reductant. Electron transfer to the 3,5-dinitrobenzoate becomes exergonic under these circumstances, and it leads to a quenching of the iridium emission. The luminescence titration curves in Fig. 1 indicate that this quenching only occurs with the nitro-substituted benzoate (gray filled circles). For unsubstituted benzoate, by contrast, the emission intensity stays essentially constant (open circles), indicating that this anionic species is not sufficiently oxidizing, i.e. excited-state electron transfer is endergonic in this case. A doubly methylated iridium complex (Fig. 1, lower left-hand side) was investigated in order to assess the importance of the hydrogen bonds in mediating electron transfer to the 3,5-dinitrobenzoate acceptor. This methylated complex has essentially identical photoredox properties as the non-methylated parent complex, but it cannot form hydrogen bonds. When titrated with dinitrobenzoate, its luminescence intensity decreases (open squares), but much less readily than for the non-methylated complex (gray filled circles). This result shows that the hydro- 


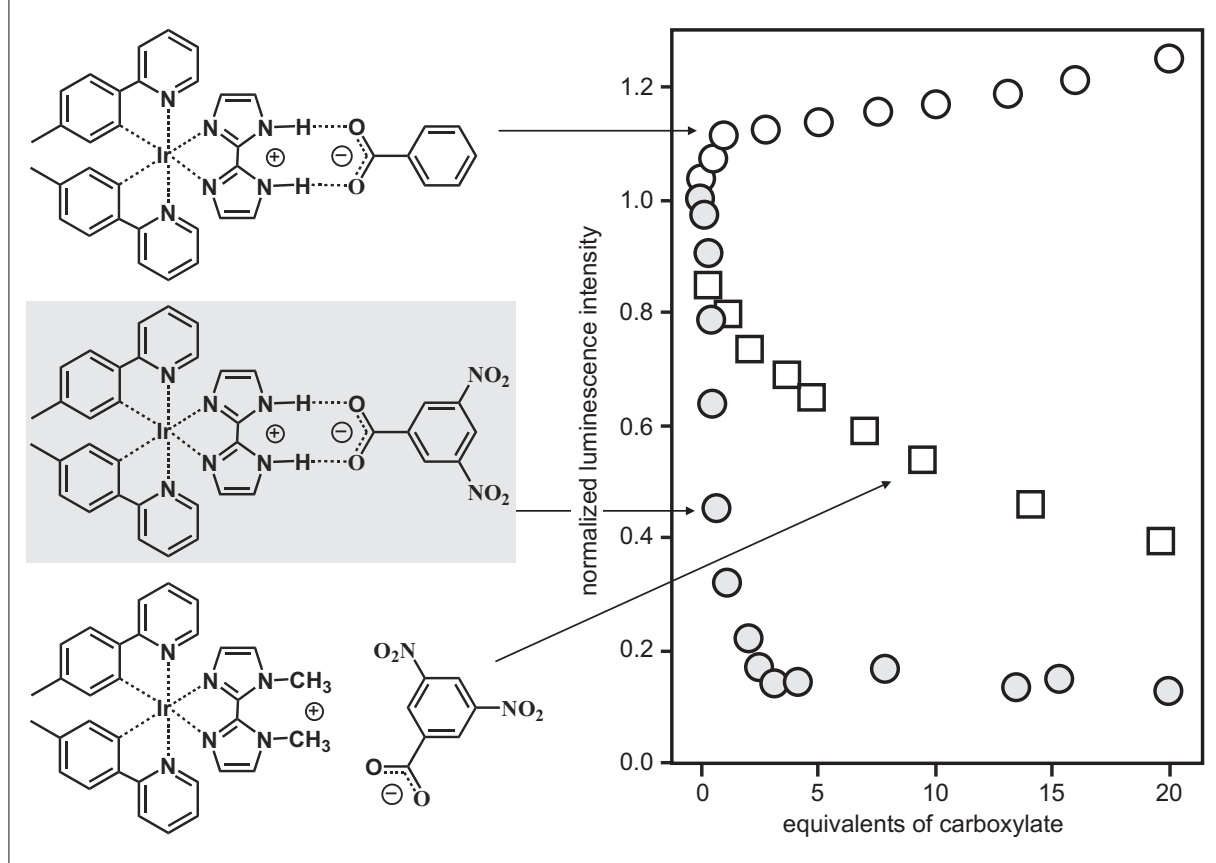

Fig. 1. Cation-anion pairs investigated in the context of proton-coupled electron transfer. ${ }^{[3]}$ The hexafluorophosphate salts of the two cyclometalated iridium complexes (at zero equivalents of carboxylate added) are strongly luminescent. Upon addition of 3,5-dinitrobenzoate, this emission is quenched (right part), whereby the extent of quenching depends on whether hydrogen-bonds can be formed between the cation and the anion (gray filled circles) or not (open squares). Redox-inactive unsubstituted benzoate also forms hydrogen-bonds, but it does not quench the luminescence (open circles).

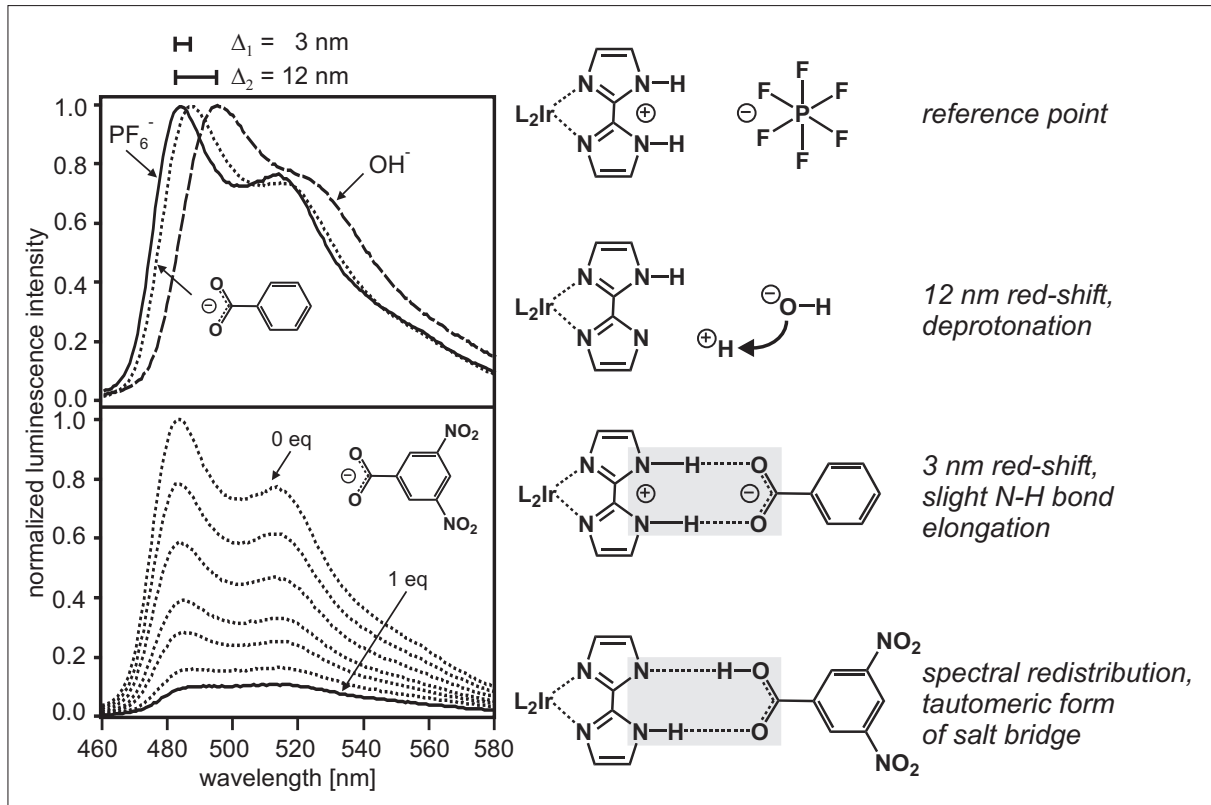

Fig. 2. The luminescence spectra of the non-methylated iridium complex are sensitive to the chemical nature of the anion (left). ${ }^{[3]}$ The spectral changes are interpreted in terms of varying proton densities at the outer biimidazole nitrogen atoms as a function of the anion (right).

gen bonds have an important impact on the efficiency of the photoinduced electron transfer process.

As far as the non-methylated iridium complex is concerned, it turns out that not only the luminescence intensity but also the emission spectrum itself is sensitive to the chemical nature of the anion (Fig. 2). [3] When hexafluorophosphate (upper panel, solid trace) is replaced by unsubstituted benzoate, the emission band redshifts by $3 \mathrm{~nm}$ (upper panel, dotted trace). Upon addition of one equivalent of the strong base tetrabutylammonium hydrox- ide to the hexafluorophosphate salt of this complex, a red-shift of $12 \mathrm{~nm}$ is observed (upper panel, dashed trace). From this we learn that large red-shifts $(12 \mathrm{~nm})$ signal the release of a full proton from the complex, whereas small red-shifts $(3 \mathrm{~nm})$ are indicative of only partial release of proton density as a result of hydrogen-bond formation to the benzoate. When the anion is dinitrobenzoate, we observe the abovementioned quenching (Fig. 2, lower panel), but there is also a spectral redistribution of the luminescence intensity which is much more important than for the unsubstituted (redox-inactive) benzoate. This intensity redistribution is consistent with a greater extent of proton delocalization away from the iridium complex towards the dinitrobenzoate than is the case for unsubstituted benzoate. It is plausible that the emissive species in the iridium-dinitrobenzoate couple is a tautomeric form of the salt-bridge formed with unsubstituted benzoate (Fig. 2, lower right, shaded areas). In other words, electron transfer is accompanied by a shift of proton density to the electron acceptor, and the iridium-dinitrobenzoate couple can be considered as a simple model system for proton-coupled electron transfer. An attractive feature of this model system is that both electron and proton transfer can be monitored easily: Information on the electron transfer step is in luminescence intensities, information on proton transfer is in spectral shifts and intensity redistributions. ${ }^{[3]}$

\section{Long-range Electron Transfer}

Our efforts in this research area started with the investigation of the rigid rod-like donor-bridge-acceptor molecules shown in Fig. 3. ${ }^{[4]}$ The donor is a phenothiazine (PTZ) molecule in all cases, the bridges are either variable-length oligo- $p$-xylenes $\left(\mathrm{xy}_{\mathrm{n}}\right)$ or oligo- $p$-phenylenes $\left(\mathrm{ph}_{\mathrm{n}}\right)$, and the acceptors are $\mathrm{d}^{6}$ metal diimines. We use a modular, iterative strategy for the synthesis of these molecules, whereby Suzuki- and Stille-type C-C couplings play an important role (Fig. 4). ${ }^{[5]}$ The latter figure also illustrates the fact that our chemistry involves more than just photons, protons, and electrons. The absorption spectra in Fig. 3 show that there are important differences between the xylene and the unsubstituted phenylene bridges. Upon phenylene bridge lengthening, their UV absorptions undergo a strong red-shift (top panel), whereas the xylene-bridged molecules exhibit much less length-dependent absorption properties (middle and bottom panels). Evidently, steric hindrance between adjacent bridging xylyl units forces them into a pronounced out-of-plane equilibrium conformation, thereby impeding significant $\pi$-conjugation 


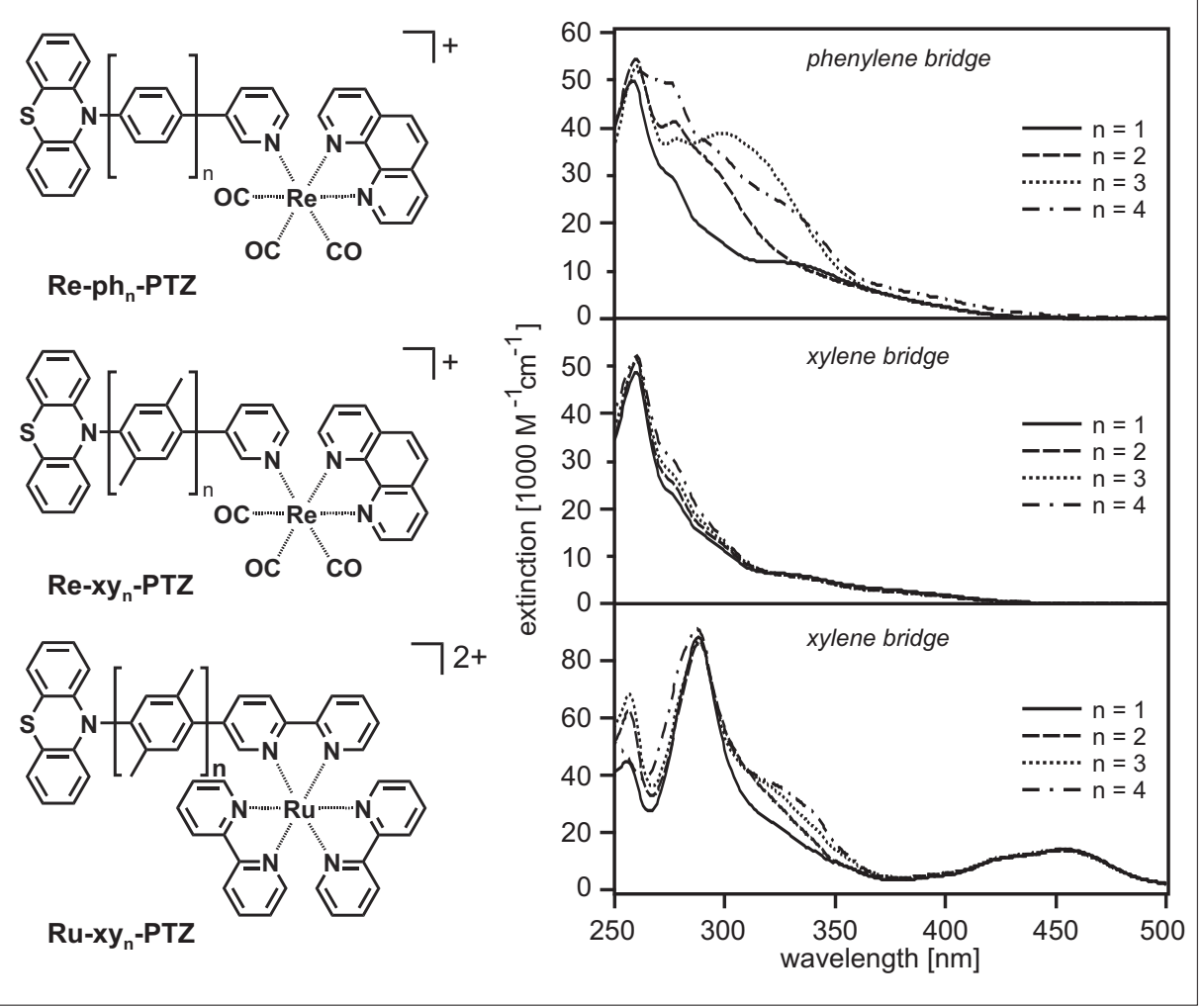

Fig. 3. Left: Donor-bridge-acceptor molecules investigated in the context of long-range charge tunneling ${ }^{[4,6,7]}$ Right: Optical absorption spectra of these dyads as a function of bridge length in dichloromethane solutions.

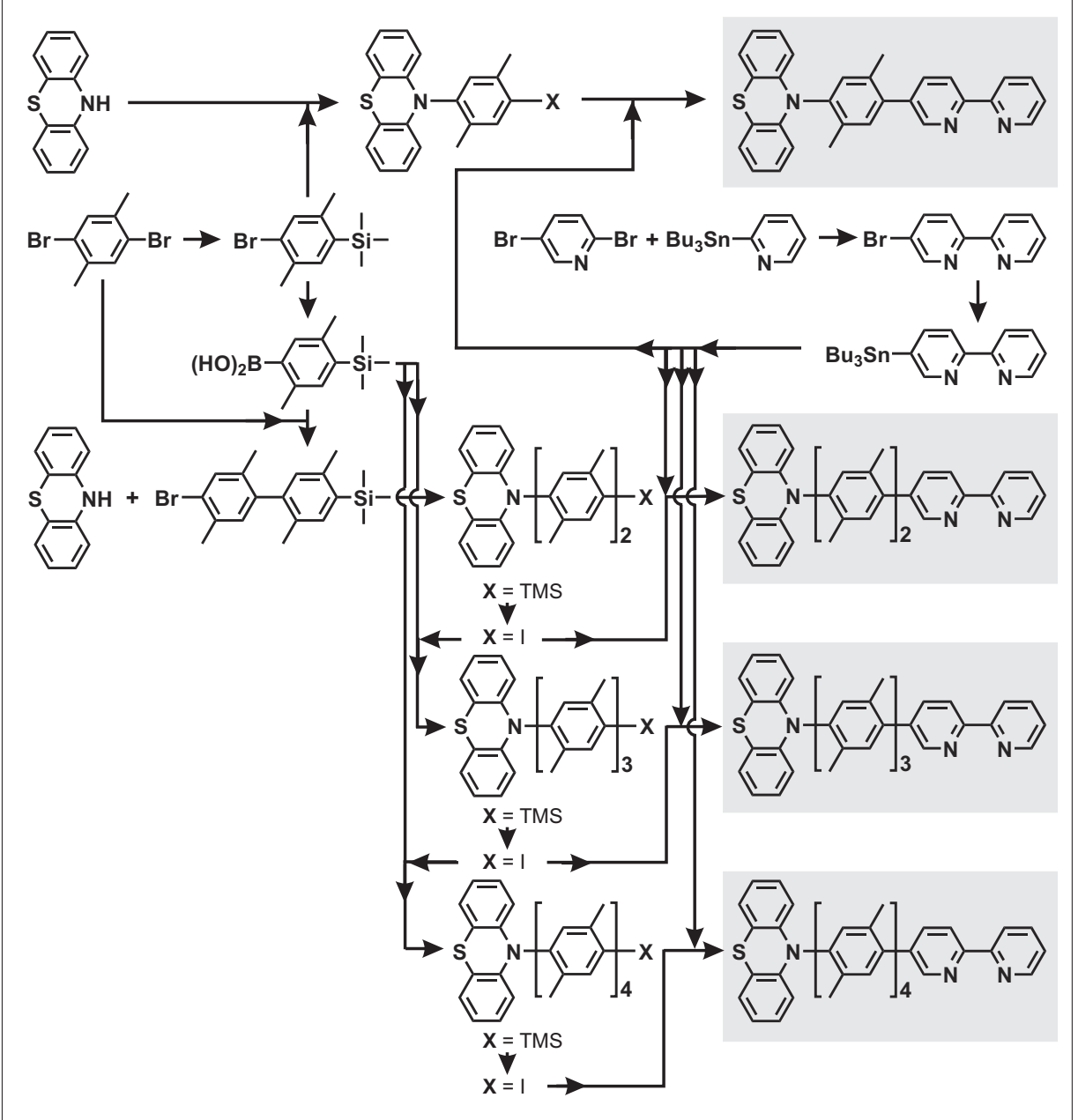

Fig. 4. Synthesis of the donor-bridge entities with 2,2'-bipyridine ligands. ${ }^{[7]}$ The synthesis of the pyridine ligands shown in Fig. 3 follows a similar strategy. ${ }^{[4,5]}$ of the oligo- $p$-xylene bridges. In the unsubstituted oligo- $p$-phenylenes, steric hindrance is less important and $\pi$-conjugation increases with increasing length, hence the red-shift in absorption. The xylyl bridges with their nearly length-independent HOMO-LUMO energy gaps are particularly useful for our investigations of barrier effects on long-range charge tunneling processes because in these molecules we can expect the barrier height to be nearly bridge length-independent. Such investigations are not possible with oligo- $p$-phenylenes since in these bridges both barrier width and barrier height change as a function of length. ${ }^{[6]}$

Photoexcitation of the metal complexes from Fig. 3 promotes them into metal-toligand charge transfer (MLCT) states in which they are at the same time better oxidants and reductants than in their electronic ground states. In the case of the rhenium(I) dyads, this triggers directly electron transfer from the phenothiazine donor to the MLCT-excited photosensitizer. ${ }^{[4]}$ The photoexcited ruthenium(II) center, by contrast, is not sufficiently oxidizing, and we must apply a bimolecularflash-quench technique to generate a more oxidizing $\mathrm{Ru}$ (III) species if we want to observe intramolecular PTZto-Ru electron transfer (Fig. 5): [7] Methylviologen $\left(\mathrm{MV}^{2+}\right)$ is used for this purpose, and since this potent acceptor is present at $\sim 500$-fold molar excess, $\mathrm{Ru}(\mathrm{III})$ is generated already within the $10 \mathrm{~ns}$ duration of the excitation laser pulse. Subsequent recombination between oxidized Ru- $\mathrm{xy}_{\mathrm{n}}-\mathrm{PTZ}$ dyad and reduced methylviologen $\left(\mathrm{MV}^{+}\right)$ occurs on timescales longer than $40 \mu \mathrm{s}$, which leaves us with a time window ranging from $10 \mathrm{~ns}$ to $\sim 40 \mu$ s for observation of intramolecular PTZ-to-Ru(III) electron transfer (Fig. 5, gray shaded area). The left part of Fig. 6 shows a transient absorption spectrum measured in this time window on the $\mathrm{Ru}-\mathrm{xy}_{4}-\mathrm{PTZ}$ dyad $\left(\mathrm{CH}_{3} \mathrm{CN}\right.$ solution, solid trace). There are intense positive signals at $\sim 400 \mathrm{~nm}$ and $\sim 600 \mathrm{~nm}$ due to reduced methylviologen $\left(\mathrm{MV}^{+}\right)$. At 520 $\mathrm{nm}$ there is a positive signal that is only observed in Ru-Xy -PTZ dyads, but not for the $\mathrm{Ru}\left(2,2^{\prime} \text { '-bipyridine }\right)_{3}{ }^{2+}$ reference complex (dotted trace). This signal is due to phenothiazine radical cation $\left(\mathrm{PTZ}^{+}\right)$. The negative signal at $450 \mathrm{~nm}$ is a so-called MLCT bleach which is characteristic of $\mathrm{Ru}(\mathrm{III})$. Thus, there are two spectroscopic observables for investigation of the dynamics of intramolecular PTZ-to-Ru(III) electron transfer: $\mathrm{PTZ}^{+}$formation at $520 \mathrm{~nm}$ and $\mathrm{Ru}(\mathrm{III})$ disappearance at $450 \mathrm{~nm}$. The temporal evolution of these two signals in the Ru- $\mathrm{xy}_{4}-\mathrm{PTZ}$ dyad is shown in the right part of Fig. 6. The same experiments can be performed with the shorter congeners with bixylyl and trixylyl bridges, whereby the distance dependence of PTZ-to-Ru(III) 

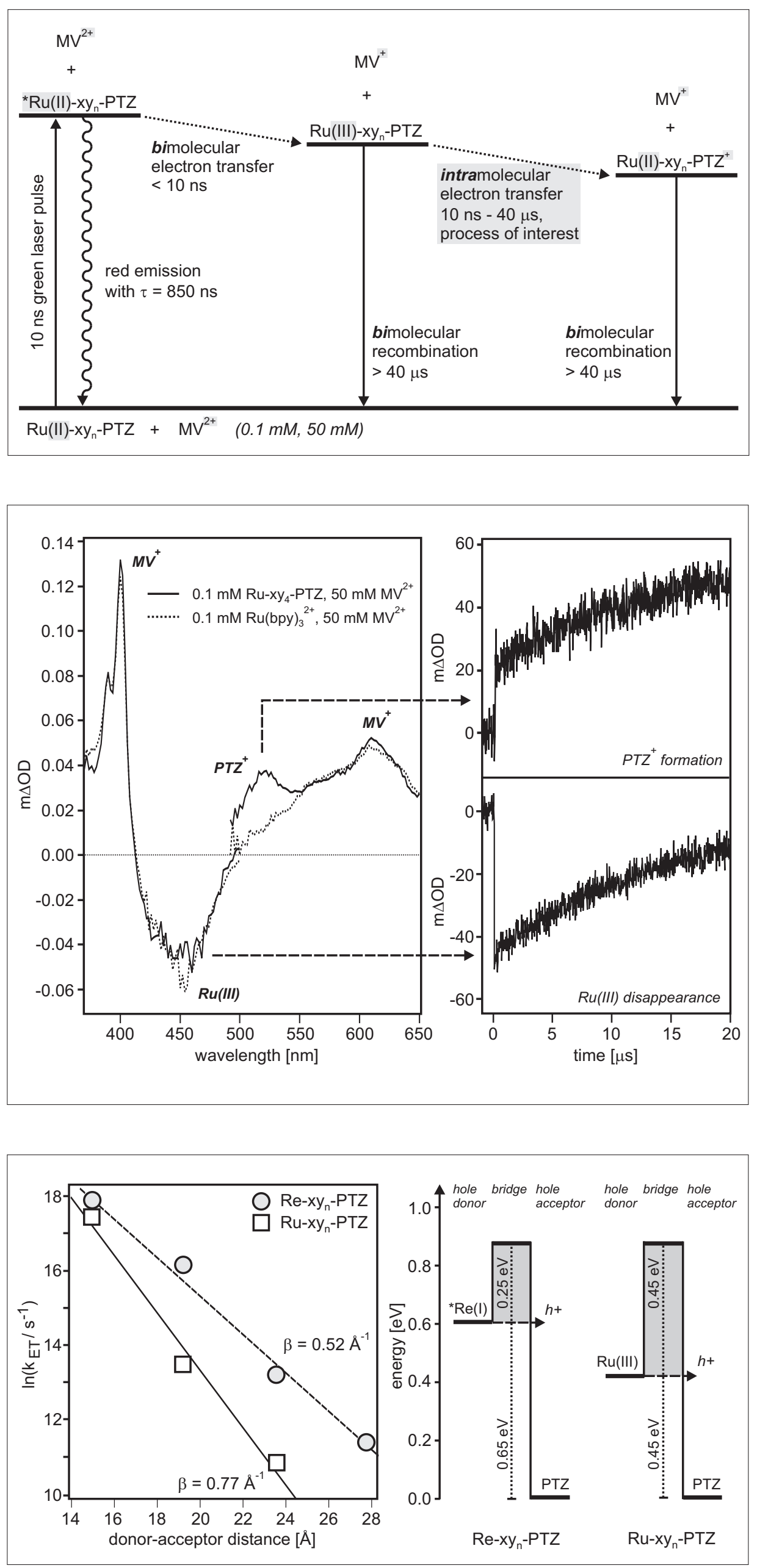

Fig. 5. Inter- and intramolecular photoinduced processes observed when investigating 0.1 $\mathrm{mM}$ acetonitrile solutions of the ruthenium dyads from Fig. 3 in presence of a 500-fold excess of methylviologen $\left(\mathrm{MV}^{2+}\right){ }^{[7]}$

Fig. 6. Left: Transient absorption spectra measured on $0.1 \mathrm{mM}$ acetonitrile solutions of the Ru-xy ${ }_{4}-\mathrm{PTZ}$ dyad (solid line) and the $\mathrm{Ru}\left(2,2^{\prime} \text {-bipyridine }\right)_{3}{ }^{2+}$ reference complex (dotted line) in presence of $50 \mathrm{mM}$ methylviologen $\left(\mathrm{MV}^{2+}\right){ }^{[7]}$ The data was acquired using a time gate ranging from $20 \mu$ s to 100 $\mu$ s after excitation at $532 \mathrm{~nm}$ (for data below $500 \mathrm{~nm}$ ) and $457.9 \mathrm{~nm}$ (for data above 500 $\mathrm{nm}$ ) with laser pulses of $10 \mathrm{~ns}$ duration. Right: Temporal evolution of the phenothiazine radical cation signal at $520 \mathrm{~nm}$ (upper right) and the ruthenium MLCT bleach at $450 \mathrm{~nm}$ (lower right) in the Ru-xy ${ }_{4}-\mathrm{PTZ}$ dyad. The pump wavelength was $532 \mathrm{~nm}$ in both cases.
Fig. 7. Left: Distance dependence of the rate constants for hole tunneling across oligo- $p$ xylene bridges in the rhenium and ruthenium dyads from Fig. 3. ${ }^{[4,7]}$ Right: Energy level diagram for hole tunneling in these dyads. Energies were estimated from redox potentials and are given relative to the phenothiazine potential. ${ }^{[7]}$ 
electron transfer rates can be investigated. This distance dependence is found to be exponential (Fig. 7, left, open squares) with an attenuation factor $\beta=0.77 \AA^{-1}{ }^{[7]}$ Interestingly, from time-resolved luminescence experiments on analogous rhenium(I)-xylene-phenothiazine dyads (Fig. 3, Re- $\mathrm{xy}_{\mathrm{n}}-$ PTZ), ${ }^{[4]}$ we find $\beta=0.52 \AA^{-1}$ (Fig. 7, left, gray filled circles), i.e. the same bridge is found to mediate electron transfer with two markedly different efficiencies.

The explanation for this behavior are the different barriers associated with charge tunneling in the rhenium and ruthenium dyads. In both cases a hole tunneling mechanism is prevalent for energetic reasons. ${ }^{[7]}$ Based on the redox potentials for the photogenerated $\mathrm{Ru}(\mathrm{III})$ and photoexcited *Re(I) hole donors, $p$-xylene bridges, and phenothiazine hole acceptors, it is possible to estimate the magnitudes of the two barriers (Fig. 7, right): ${ }^{[7]}$ Hole tunneling from $\mathrm{Ru}(\mathrm{III})$ to PTZ is associated with a barrier of $\sim 0.45 \mathrm{eV},{ }^{*} \operatorname{Re}(\mathrm{I})$-to-PTZ hole tunneling only with a barrier of $\sim 0.25 \mathrm{eV}$. It is possible to estimate effective tunneling barriers $\Delta \mathrm{E}_{\text {eff }}$ from experimental $\beta$-values based on an expression that emerges from a combination of superexchange theory and a mathematical expression describing the probability for a particle of mass $m$ for tunneling through a square-wave potential energy barrier:[7,8]

$$
\Delta E_{\text {eff }}=\left(\frac{\hbar^{2}}{8 \cdot m}\right) \cdot \beta^{2}
$$

For the electron, the prefactor in Eqn. (1) is $0.952 \mathrm{eV} \cdot \AA^{2}$, yielding $\Delta \mathrm{E}_{\text {eff }}=0.55$
eV for Ru-xy $-\mathrm{PTZ}\left(\beta=0.77 \AA^{-1}\right)$ and $\Delta \mathrm{E}_{\text {eff }}$ $=0.26 \mathrm{eV}$ for Re-xy -PTZ $\left(\beta=0.52 \AA^{-1}\right)$. Both of these effective barriers are close to the barriers estimated above from redox potentials, thereby supporting our interpretation of the different $\beta$-values in Fig. 7 in terms of different tunneling barrier heights.

This work demonstrates that a given molecular bridge can mediate long-range charge tunneling with varying efficiencies, depending on the potentials of the attached redox partners. It is therefore conceivable that a given bridge may exhibit significantly different distance dependences for photoinduced charge separation (an energy-storing electron transfer) and thermal charge recombination (an energy-wasting electron transfer). This may represent a way to discriminate between desired and undesired electron transfer reactions. Research in that direction is underway.

\section{Summary}

Our key research interests are phototriggered proton-coupled electron transfer reactions and long-range charge tunneling processes, with particular emphasis on the dynamics of these reactions. For this purpose, organic and coordination chemical synthetic work is complemented by physical-inorganic spectroscopic studies. Our research is relevant in the greater contexts of light-to-chemical energy conversion, small molecule activation, and molecular electronics.

\section{Acknowledgments}

We thank the Swiss National Science Foundation for sponsoring the presented research and a related project with two independent grants. The Department of Inorganic, Analytical and Applied Chemistry and the chemistry section at the University of Geneva are thanked for excellent hosting and additional financial support of the newly created group. The Swiss State Secretariat for Education and Research is acknowledged for financing a postdoctoral position. The Société Académique de Genève and the Fondation Ernst \& Lucie Schmidheiny are thanked for financial contributions to the acquisition of laboratory equipment. We are grateful to Prof. Andreas Hauser for granting us access to his laser facilities. The corresponding author wishes to express his gratitude to his former mentors Hans U. Güdel, Harry B. Gray, and Jean-Pierre Sauvage for what he could learn from them.

Received: December 15, 2008

[1] R. Eisenberg, H. B. Gray, Inorg. Chem. 2008, 47, 1697.

[2] J. B. Howard, D. C. Rees, Proc. Natl. Acad. Sci. U. S. A. 2006, 103, 17088.

[3] J. C. Freys, G. Bernardinelli, O. S. Wenger, Chem. Commun. 2008, 4267.

[4] D. Hanss, O. S. Wenger, Inorg. Chem. 2008, 47, 9081.

[5] M. E. Walther, O. S. Wenger, Dalton Trans. 2008, 6311.

[6] O. S. Wenger, Coord. Chem. Rev. published online, doi: 10.1016/j.ccr.2008.10.010.

[7] D. Hanss, O. S. Wenger, Inorg. Chem. 2009, 48, 671.

[8] O. S. Wenger, Chimia 2007, 61, 823. 